\title{
PERCEPTION OF APPROACHING MOTORCYCLES BY DISTRACTED DRIVERS MAY DEPEND ON AUXILLARY LIGHTING TREATMENTS: A FIELD EXPERIMENT
}

\author{
James Jenness, Richard Huey, Shawn McCloskey, Jeremiah Singer, \\ Jeremy Walrath, Elisha Lubar, \& Neil Lerner \\ Westat \\ Rockville, Maryland, USA \\ Email: jamesjenness@westat.com
}

\begin{abstract}
Summary: A field experiment was conducted in daylight with 32 participants to determine whether left turning drivers' gap acceptance in front of approaching motorcycles depends on the motorcycle's forward lighting treatment. Five experimental lighting treatments including a modulated high beam headlamp, or the low beam headlamp plus pairs of low-mounted auxiliary lamps, high-mounted auxiliary lamps, both high- and low-mounted auxiliary lamps, or low-mounted LED lamps were compared to a baseline treatment with only the low beam headlamp illuminated. Participants viewed the approaching traffic stream (including the motorcycle) on an active roadway and indicated when it would be safe (and not safe) to initiate a left turn across the opposing lanes. Participants also shared their attention with a secondary visual distraction task that took their eyes off the forward roadway. Participants did not know that the purpose of the study was to measure their responses to approaching motorcycles. Based on participants' indications of the last safe moment to turn, the mean temporal safety margin provided to the approaching motorcycle did not differ significantly between any of the experimental lighting treatments and the baseline treatment. However, having either low-mounted auxiliary lamps or modulated high beam lamps on the motorcycle significantly reduced the probability of obtaining a potentially unsafe short safety margin as compared to the baseline lighting treatment. Overall, the results suggest that enhancing the frontal conspicuity of motorcycles with lighting treatments beyond an illuminated low beam headlamp may be an effective countermeasure for daytime crashes involving right-of-way violations.
\end{abstract}

\section{OBJECTIVES}

The annual number of motorcycle rider fatalities in the United States has more than doubled from 2294 in 1998 to 5290 in 2008 (National Highway Traffic Safety Administration, 2010). Over the same period, the total number of traffic fatalities has remained relatively stable. Many multi-vehicle motorcycle crashes involve right-of-way violations with another vehicle turning in front of, or crossing the path of an on-coming motorcycle (Longthorne, Varghese, \& Shankar, 2007) and the driver of the other vehicle often claims not to have seen the motorcycle (Hurt, Ouellet, \& Thom, 1981). Improving the frontal conspicuity of motorcycles may reduce the occurrence of these types of crashes. Daytime use of illuminated lamps on motorcycles has been shown to increase their conspicuity (Wulf, Handcock, \& Rahimi,1989; Smither \& Torrez, 2010). 
For many years, motorcycles sold in the U.S. have been equipped with at least one low beam headlamp that is illuminated automatically when the engine is started. The purpose of this study was to determine whether additional frontal lighting will enhance motorcycle conspicuity during the daytime. The primary objective was to determine whether five different forward lighting treatments differ from a baseline treatment with a single low beam headlamp in helping motorists notice and respond appropriately to approaching motorcycles on an active roadway.

\section{METHODS}

\section{Participants}

Researchers recruited 32 licensed adult drivers to participate individually in a field study that was advertised as a study of drivers' eye movements and perception of approaching vehicles. Participants were not informed that the specific purpose of the study was to examine their responses to approaching motorcycles. Participants included an equal number of men and women. The mean age of participants was 39 years (range 19 to 67). Potential participants were screened with regard to driving experience with different types of vehicles including motorcycles. No experienced motorcycle riders or people who have motorcycle riders in their immediate family were selected to participate. Participants were tested with a Snellen eye chart to ensure that they had binocular acuity of 20/40 or better.

\section{Field Site}

The study was conducted on Muddy Branch Road, a four-lane divided highway in Gaithersburg, Maryland. The posted speed limit was $45 \mathrm{mph}$. The road had a 30-foot wide grass median with guard rails that provided the site with some protection from vehicle intrusions. The research vehicle, a Chevrolet Equinox SUV, was parked in the center of the median for the study sessions. The vehicle faced north and during data collection the participant sat in the driver's seat and observed oncoming southbound traffic (Figure 1). The participant's eye position was approximately 35 inches higher than the adjacent roadway.

All experimental sessions were conducted between 11:30 AM and 2:00 PM so that the angle of the sun would be high and consistent between participants. All sessions were conducted in clear weather with the exception of one, which was conducted partially in light rain.

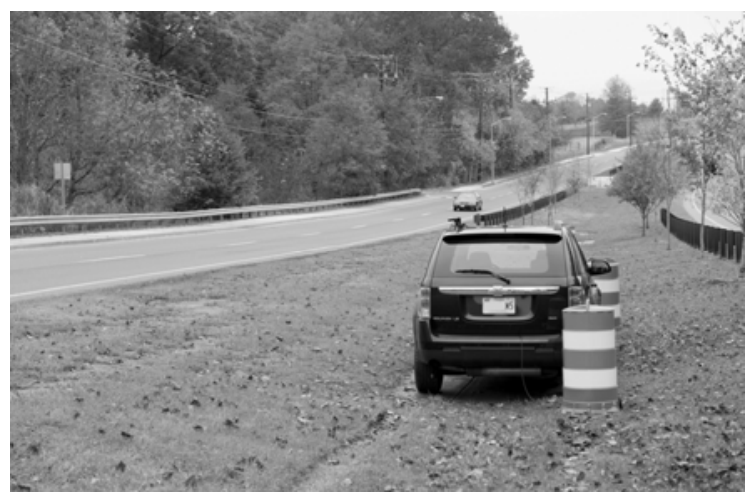

Figure 1. View of study site and research vehicle looking north toward approaching traffic 


\section{Apparatus}

Motorcycle and rider. The motorcycle used for this study was a 2007 Honda Shadow Spirit with a 750cc engine and stock exhaust pipes. The motorcycle's normally illuminated amber clearance lamps (which also serve as turn signals) were disabled except when the turn signal function was activated. The motorcycle rider was dressed in black and grey armored clothing and a full-face helmet that was matte black.

Auxiliary lamps and related equipment. Lighting treatments were selected primarily to improve conspicuity of the motorcycle as seen by other drivers rather than providing additional illumination of the forward roadway for the motorcycle rider. An important consideration was to test relatively low-power lamps because the electrical systems of many motorcycles are not designed to accommodate the high power requirements of multiple additional lamps. Despite the relatively low power requirements of the lamps used, the luminous intensities fell with the range recommended by Rumar (2003) for daytime running lights. The experimental lighting treatments included:

- $\quad \mathrm{HA}=$ One pair of auxiliary bullet lamps was mounted with lamp centers 40.5 inches above the ground, near the handle bars. The centers of the right and left lamps were 15 inches apart. The lamp housings incorporated Philips 12 volt, 20 watt, quartz-halogen incandescent flood lamps with built-in $36^{\circ}$ beam angle parabolic reflectors (MR-16 form factor).

- $\quad$ LA $=$ A second identical pair of auxiliary bullet lamps was mounted near the front forks with lamp centers 21 inches above the ground. The centers of the left and right lamps were 15.6 inches apart.

- $\quad$ LHA = Both sets of low-mounted and high-mounted auxiliary lamps described above were illuminated.

- $\quad$ LED = One pair of Hella 12 volt LED lamps (Part No. 1004) was mounted vertically above the front axle (with the lamp centers 16.5 inches above the ground). Each of these lamps incorporated a black plastic enclosure housing six rows of two adjacent, high brightness LEDs behind a flat lens assembly (1 inch wide and 5 inches tall). Each lamp required 2 watts of power. These lamps were manufactured in New Zealand where they are used as daytime running lights for passenger vehicles.

- $\quad \mathrm{MHB}=\mathrm{A}$ headlamp modulator kit (All-in-One Headlight Modulator and Solid State "Relay" Kit, model: AioSR15H4, available from Comagination.com) provided daytime modulation of the high beam headlamp at $4 \mathrm{~Hz}$ in conformance with FMVSS 108 (Federal Motor Vehicle Safety Standards) (49 CFR Part 571.108 S7.9.4). The low beam was off when the MHB was on.

For all lighting treatments except MHB, the low beam headlamp was illuminated in addition to the auxiliary lamps. The modulated high-beam headlamp and the low-beam headlamp were powered by the motorcycles' stock battery and electrical charging system. Auxiliary lamps were powered by an independent electrical supply system and the voltage was regulated at 12.8 volts to an accuracy of $\pm 0.05 \%$. 


\section{Procedure}

Participants were told that the general purpose of the study was to evaluate drivers' eye movements while they judged gaps in approaching traffic. Accordingly, participants wore a head-mounted gaze tracking system (ASL MobileEye) during the data collection period.

The participant's primary task was to watch oncoming traffic and to indicate when they could safely make a left turn across traffic. Because there was not any actual destination road to turn left on, two red traffic cones were set up $5.5 \mathrm{~m}$ apart from one another, on the sidewalk across the street to the participant's left and parallel to the road. The participant was told to imagine that the gap between the cones was a road that they could turn on. To indicate when they could start turning, the participant was instructed to press and hold a button and to then release the button as soon as he or she was no longer able to initiate a safe turning maneuver. When making turning judgments, the participant was instructed to imagine that he or she was waiting in paved, level turn lane rather than the grassy median.

The participant was also given a secondary task to perform while making turning judgments. A small white light was mounted atop the leftmost traffic cone across the street. The light turned on at random intervals between 12 and 24 seconds. The participant was instructed to press a large button attached to their left leg every time the light turned on. The location of the light, to the left of the participant, required the participant to glance away from oncoming traffic. The participant practiced making left turn judgments and simultaneously monitoring the secondary task light until he or she was comfortable with the two tasks.

The motorcyclist entered the road far enough upstream to be able to reach traffic speed before entering the participant's field of view. Rather than traveling at a fixed speed on every pass, the motorcyclist attempted to ride at the approximate speed of other traffic. He also attempted to leave a large enough gap ahead of him so that the participant would feel comfortable making a left-turn ahead of the motorcycle, and he also tried to avoid being passed by other vehicles as he approached the study site. As anticipated, these strategies led to some speed variability of the motorcycle on different passes.

The motorcyclist rode past each participant at least six times; once with each of six lighting conditions presented in predefined, quasi-random order that was counterbalanced across participants. The motorcycle passed the participant once every four or five minutes, on average. On some of these occasions another vehicle entered the traffic stream from a side road, or a speeding vehicle passed the motorcycle before it reached the study site. All such "busted" trials were repeated at the end of the session.

\section{RESULTS}

\section{Safety Margin for Turning Left}

In this study "safety margin" was defined as the elapsed time between the moment that the participant released the "turn button" to indicate that it was no longer safe to initiate a left turn, 
and the moment when the motorcycle arrived at the conflict point. This was the point where the two vehicles’ paths would have intersected if a left turn maneuver actually had been carried out.

A key research question was to determine if participants provided a greater safety margin to an approaching motorcycle that had various enhanced forward lighting treatments as compared to the baseline condition where the motorcycle had only the low beam headlamp illuminated. A second key research question was to determine whether auxiliary lighting treatments would reduce the number of potentially unsafe short safety margins accepted by participants. Although data were obtained for 32 participants and 6 lighting treatments (total = 192 trials), nine of the trials were invalid, and were excluded from further analysis.

The distribution of safety margins for approaching motorcycles had a mean of 4.97 seconds and standard deviation of 1.91 seconds. Approximately 25 percent of the measurements were less than 3.44 seconds and 25 percent of the measurements were greater than 6.35 seconds. Researchers noticed that some participants tended to be consistently more conservative than others in their judgments about when it was safe to turn. All analyses reported here account for the repeated-measures design of the study and the tendency for data to be clustered by participant.

On each trial the motorcycle's average speed was calculated based on frame-by-frame analysis of video data. Motorcycle speed between an upstream reference point and a point near the participant's vehicle varied between trials. The distribution of motorcycle speeds had a mean of $43.8 \mathrm{mph}$ and a standard deviation of $2.7 \mathrm{mph}$.

To determine whether any of the experimental lighting treatments were associated with significantly longer safety margins than the baseline condition, safety margins were modeled using the SAS Proc Mixed procedure. The model estimated effects for lighting conditions and motorcycle speed and controlled for data clustered within participants. None of the model coefficients for lighting treatments and motorcycle speed were statistically significant. The same statistical procedure was used to fit a second model to the data. This model included motorcycle speed, lighting treatments, and all of the lighting treatment by motorcycle speed interactions. None of these effects were statistically significant.

\section{Short Safety Margins}

From the safety perspective, it is most important to examine whether the various experimental lighting treatments were more or less likely than the baseline condition to be associated with short (unsafe) safety margins. If participants were actually making left turns in front of the motorcycle, initiating the turn with only a few seconds remaining before the motorcycle's arrival at the conflict point may lead to a collision or may necessitate an emergency maneuver by one or both vehicles to avoid a collision. Because the study took place at a location where there was no cross street or driveway, it was not possible to measure how much time would be required for the research vehicle to actually complete a left turn from its starting location. Therefore, for analysis purposes, all trials where the measured safety margin fell within the first quartile (less than 3.44 seconds) were defined as short safety margins. Based on researchers' judgment of the study site, it is likely that left turns initiated while the motorcycle was less than 3.44 seconds away would 
have resulted in the vehicles passing in close proximity of each other and that such turns may have required the motorcycle rider to brake or perform another evasive maneuver to avoid a collision.

Cumulative frequency distributions for safety margins obtained with each of the different lighting treatments are shown in Figure 2. Note that the midpoints of the distributions are similar but that the distributions for experimental lighting treatments (thin lines) diverge from the distribution for the baseline treatment (thick line) at short (and at long) safety margins. Also note that for safety margins less than 4 seconds, all of the experimental lighting treatment distributions fall below the distribution for the baseline treatment.

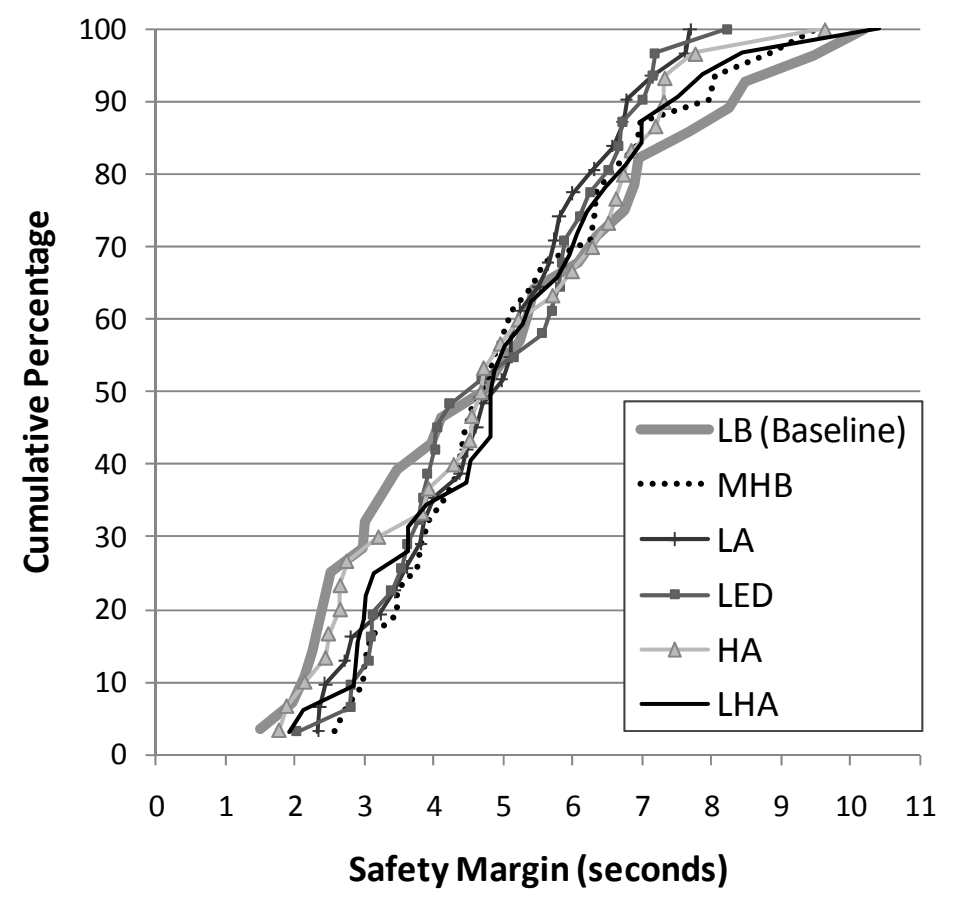

Figure 2. Cumulative frequency distributions of safety margins for motorcycle lighting treatments

The greatest proportion of valid trials that resulted in the participant giving a short safety margin occurred for the baseline condition $(10 / 28=35 \%)$ and the smallest proportion of trials with short safety margins occurred with the MHB treatment $(5 / 31=16 \%)$ and with the LA treatment $(6 / 31$ $=19 \%$ ). Other lighting treatments resulted in 23\% (LED), 25\% (LHA), and 30\% (HA) of trials with short safety margins.

To test the statistical significance of these observed differences, the probability of obtaining a short safety margin was modeled using a logistic regression model implemented with the SAS GENMOD procedure. This model specified a binomial underlying distribution for the outcome variable and a logit link function. The binary outcome variable (short safety margin versus not a short safety margin) was parameterized so that the model predicted the probability of obtaining a short safety margin based on lighting treatments and motorcycle speed. A repeated measures (subject) effect was included to account for data clustered by participant. The model used GEE (Generalized Estimating Equations) for parameter estimation. 
Based on the model's parameter estimates for lighting treatments and motorcycle speed, motorcycle speed was not a statistically significant predictor of short safety margins. However, parameter estimates for LA and MHB were statistically significant $(p<.05)$ and the parameter estimate for LHA nearly reached statistical significance $(p=.06)$. Negative values estimated for these parameters suggest that having either LA or MHB treatments on the motorcycle reduced the probability of a short safety margin as compared to the baseline condition. Although they did not reach statistical significance, the estimated effects for the other experimental treatments (LHA, LED, HA) also were negative.

\section{CONCLUSIONS}

The results of this study suggest that at least some of the lighting treatments tested have the potential to reduce the occurrence of potentially unsafe short safety margins imposed on approaching motorcycles by partially distracted left-turning drivers. As compared to the baseline lighting treatment, participants viewing a motorcycle with LA or MHB treatments were less likely to indicate that it remained safe to turn across the motorcycle's path when the motorcycle’s time until arrival had decreased to less than 3.44 seconds.

Enhancing the forward lighting on motorcycles during the daytime may be effective for reducing the probability that drivers will turn in front of the motorcycle with an unsafe short safety margin. Potentially, this may reduce crash rates for left-turn across path scenarios involving drivers who unknowingly violate the motorcyclist's right-of-way.

\section{ACKNOWLEDGMENTS}

Funding for this research was provided by the National Highway Traffic Safety Administration under contract DTNH22-05-D-01002. The views expressed in this work are those of the authors and do not necessarily represent official NHTSA policy.

\section{REFERENCES}

Hurt, H.H., Ouellet, J.V. \& Thom, D.R. (1981). Motorcycle Accident Cause Factors and Identification of Countermeasures: Volume 1 (Technical Report No. DOT HS-5-01160). Los Angeles: University of Southern California, Traffic Safety Center.

Longthorne, A., Varghese, C., and Shankar, U. (2007). Fatal Two-Vehicle Motorcycle Crashes. Report No. DOT HS 810 834. Washington DC: National Highway Traffic Safety Administration.

National Highway Traffic Safety Administration, (2010). Fatal Analysis Reporting System Encyclopedia. (Retrieved April 16, 2010 from www-fars.nhtsa.dot.gov.) Washington, DC: National Highway Traffic Safety Administration.

Rumar, K. (2003). Functional Requirements for Daytime Running Lights. Report No. UMTRI-200311, Ann Arbor: The University of Michigan Transportation Research Institute.

Smither, J.A. \& Torrez, L.I. (2010). Motorcycle conspicuity: Effects of age and daytime running lights. Human Factors, 52(3), 355-369.

Wulf, G. Handcock, P.A. \& Rahimi, M. (1989). Motorcycle conspicuity: An evaluation and synthesis of influential factors. Journal of Safety Research, 20, 153-176. 